\title{
Dénes ZARKA
}

\section{Online collaboration practice for active learning in the visual age}

\section{The rationale of Vocal project}

The diversity of the European educational culture, languages, legislation, and territorial fragmentation suggest, that educational organizations in Europe need to network and collaborate to achieve inclusive education and training services in terms of:

- diversification of learners

- the opening up of education

- the preparedness to merge international and intercultural learner groups

- provision of learning services for work-based learners

European (C)VET and higher education institutions should be digitally competent to meet these challenges and provide quality learning services, with a special focus on digitally and pedagogically competent teachers and trainers.

\section{EC "Work-based learning in Europe" policy document (2013)}

An articulated reaction to this problem set was a policy document about work-based learning. This document contains two important aspects:

- to identify the need to increase opening up in educational practices to increase:

o integrated work-based learning activities

o effective and quality learning examples on open, inclusive, user-friendly environments

- teachers claim - they are challenged when embedding digital, social and intercultural competences in the curriculum, especially if the curriculum is digitalised, developed online and are aimed at diverse target groups.

\section{Collaboration and visual age?}

What is the link between digitalisation, collaboration and visual learning? To answer that, we have to answer the following questions:

What do we need for effective online collaboration? The following three aspects are evidently among the answers:

- Splitting up our concentration between different channels, like Computer system status, different parallel application messages, and the data flow of the collaborative software.

- It is also a challenge for most of us, to find orientation in a virtual environment. Finding quick orientation in time and space (on-screen) is even more demanding. If there are several chat boxes, for example, one on the computer desktop, one in FB messenger, one in the webinar room, it is not evident where to click: to read and answer the message. It is also a challenge to have a local and a remote document (shared screen) and find (visually) quickly a written expression that is discussed orally during the meeting.

- When a group of colleagues are in the deep discussion, it is always a challenge to identify the collaborators quickly, e.g. to match the voice with the person if the camera is not switched on. 
A well designed visual environment can help us. Good pictograms, moving, blinking, "dancing" icons may dramatically speed up our orientation in the virtual environment.

\section{Tools used for online collaboration}

The vocal project was designed to use MOODLE as a central collaboration tool, therefore most of the tools used in the project were Moodle basic tools, but some tools were linked out to experience Open educational practice as well. Apart from Moodle, project partners used the following applications:

- written collaboration - Google docs, wiki

- oral collaboration - Skype, Messenger

- video collaboration - BBB (Big Blue Button), AC Pro (Adobe Connect Pro)

- complex collaboration - Online workshop (Moodle)

\section{Written online collaboration}

Let us observe further the written on-line collaboration. The following visual challenges can be listed as an example:

- Identification of authors/users on-line. That case arrives when multiple editors work on an on-line document simultaneously. It is not self-explanatory, who exactly is/was writing/editing a given part of the text. Solution: profile picture, or avatar for anonymous users.

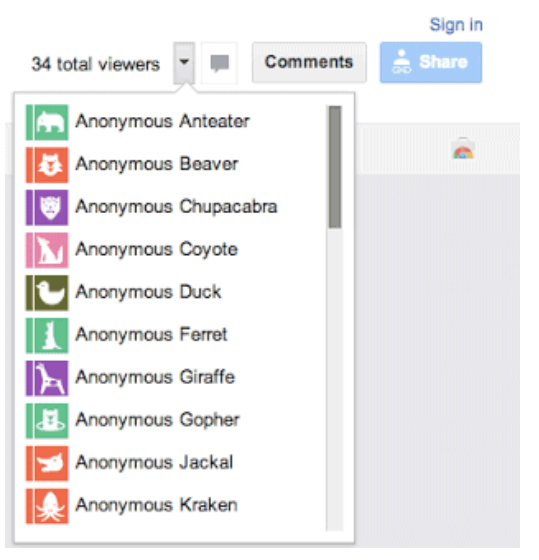

- Identification of different text types. That is the task of visually represent the different author text parts, and to organise a quick reference to the author/user. Solution: Coloured text parts.

- Identification of tools to be used. This is a less problematic, but still important visual problem: The representation of the text editing tools so that most of the authors/users understand it without going through a tutorial before using the collaborative tool. Solution: A text editing icon standard, that is well known in all text editors

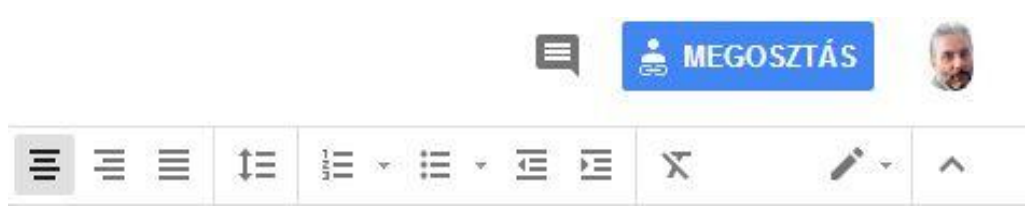


- Easy to use the system of tracking. This is a longitudinal problem of tracking the different text versions and comments as time goes by. Even a smaller collaborative author group may generate multiple layers of the shared document, and it is not easy to visually help to track those layers. Solution: The introduction of marginal notes to each modification, attached to a close text part.

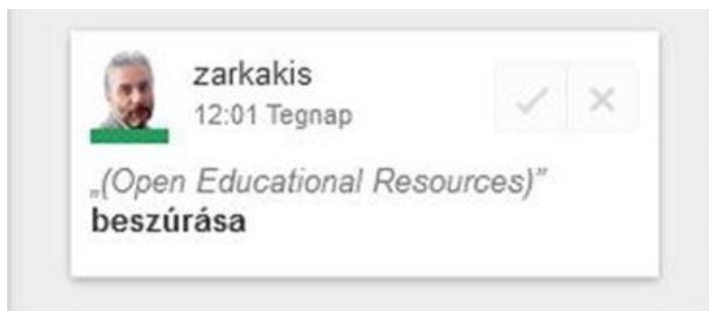

\section{Oral online collaboration}

Next common collaboration type is an oral collaboration, where we also have some challenges when we use skype, messenger, or other tools.

- The visual orientation of the main oral call features. This type of communication is originated from telephone calls in telephone (telecom) industry. Solution: Easy to understand pictograms, for the pure oral call, video call, and group call:

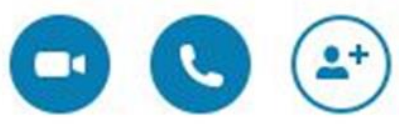

- The visual orientation of text call features (chat). During the conversation, it is possible to use different alternative channels of communication or to use other functions of the application. During the "main" communication, those parts are hidden. Solution: Function icons change their shape to signal: something has changed "behind". (Example: the smiley with orange point show a hidden incoming chat text.)

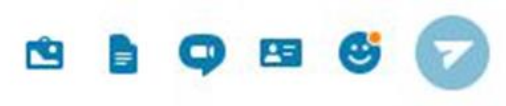

- The visual orientation of the calling process. The calling process in case of classic telephone equipment was easy, either we took the phone, dialled, and were waiting for the ringing (or engaged) tone, or our equipment rang with an easily separable tone. Now in case of an application that is partly substituting this communication have to visually and orally simulate this process, so that the users are aware of the fact that they are "really" calling someone, and to realise if the call was successful, and the other party is on-line or the call failed for different reasons (patching also the reason). It is also important to know exactly if the user is called. (Bearing in mind that many multimedia applications may be in use). Solution: Use of an extra calling panel that is visible on the desktop and following the calling process visually as well.

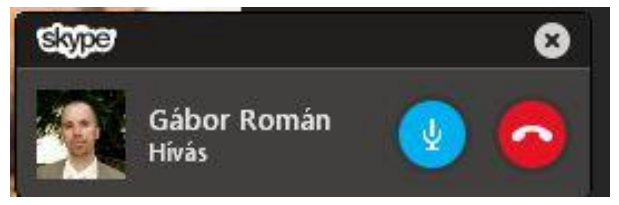


- The visual orientation of identification of calling and active parties. In case of oral online communication conference calls are much more frequently used as in case of traditional telephone systems, where it was made with a so-called "bridge". Technically it is still possible and easy to use. However, an important problem occurs (and occurred earlier for those who already used traditional teleconference systems), namely the fact that users do not recognise the actively speaking party from their voice (tone). The traditional answer to this problem was that users were moderated, and had the right to speak only in case of explicit invitation to speak by the moderator. Solution in computer systems:

- a cascade (first come first serve) walkie-talkie mode by allowing only one microphone to be active.

- a visual representation of all parties that are on-line with avatar or picture, and an emphasized frame on behalf of the active speaker (in case of multiple active microphones looping may occur).

\section{Conclusion}

In this paper, we discussed the visual elements of on-line collaboration based on the experience of VOCAL project. We could explain in detail, and give evidence, that to smoothly use on-line collaborative applications for virtual group work, it is essential that well-designed visualisation support the process. In other words: to learn on-line collaboration with given tools a visual learning process is also essential. The real learning efforts and the needs for this visual learning should be researched further in the future.

\section{References}

- Vocal project - www.vocalerasmus.eu

- Vocal learning portal - www.Ims.vocalerasmus.eu

- "Work-based learning in Europe -Renewing Traditions" - background information https://www.bibb.de/dokumente/pdf/stbpr veranstaltung 20131204 workbased learnin $\mathrm{g}$ in europe hintergrundpapier en.pdf. 